\title{
Hypertonic saline nasal irrigation and gargling as an inexpensive practical adjunctive weapon to combat asymptomatic SARS-CoV-2 infections. A case report
}

\author{
Paola Rosati $^{1 *}$, Ugo Giordano ${ }^{2}$ and Carlo Concato ${ }^{3}$ \\ ${ }^{1}$ Clinical pathways and Epidemiology Unit, Bambino Gesù Children's Hospital IRCCS, Largo S. Onofrio 4, 00165 Rome, Italy \\ ${ }^{2}$ Paediatric Cardiology and Cardiac Surgery Department, Bambino Gesù Children's Hospital IRCCS, Largo S. Onofrio 4, 00165 Rome, Italy \\ ${ }^{3}$ Virology Unit, Laboratory Department, Bambino Gesù Children's Hospital IRCCS, Largo S. Onofrio 4, 00165 Rome, Italy
}

\begin{abstract}
Background: Preventive policies and harmful lockdowns in Italy, Western Europe, the United States, as well as in other developing countries worldwide seem unable to contain the intensive care burden and deaths for severe acute respiratory syndrome coronavirus 2 (COVID-19), especially in nursing homes. Prompted by the limited evidence available, we wonder why until now only one publication (JAMA Otolaryngol Head Neck Surg doi:10.1001/jamaoto.2020.1622) has documented how repetitive nasal rinsing and gargling with hypertonic saline (a solution three to four times as salty as normotonic saline) could quickly contribute as an adjunctive cost-free weapon to current health policies for use against new coronavirus 2019 (SARS-CoV-2) infections at an early stage, also in quarantined people, and in developing countries.

Case presentation: We report the case of a 54-year-old asymptomatic SARS-CoV-2 infected physician (positive nasopharyngeal swab test) quarantined in Rome in whom hypertonic saline nasal rinsing and gargling several times a day at home induced a rapid successful response. The infected physician remained asymptomatic, and serum immunoglobulin IgG for SARS-CoV-2 tested negative two months after the infection.

Discussion and conclusions: Our case report, describing at more than two months after the SARS-CoV-2 infection negative serum IgG, seemingly supports recent evidence suggesting that asymptomatic subjects treated early with hypertonic saline solutions avoid multi-organ inflammation needing intensive care. In the current urgency state, health authorities could advocate hypertonic saline nasal rinsing and gargling as an adjunctive inexpensive procedure for SARS-CoV-2 "wash-out”. This proven, generally safe saline expedient might help as an adjunctive weapon like handwashing to combat the SARS-CoV-2 infection early in developed and developing countries, or returning as second COVID-19 waves.
\end{abstract}

\section{Background}

In vitro, irrefutable biochemical evidence shows that chloride $(\mathrm{Cl})$ ions, converted into hypochlorous acid $(\mathrm{HOCl})$, are an innate human immune mechanism mediated by phagocytes and neutrophils (non-myeloid cells) to inhibit viral replication [1,2]. Viral infections significantly increase intracellular $\mathrm{HOCl}$ [2]. In vivo, intracellular $\mathrm{Cl}^{-}$ions permit non-myeloid cell antiviral activity while salt attracts water into the airways, thus making it easier to eliminate viruses with coughing [1-3].

A simple and inexpensive way to increase $\mathrm{Cl}^{-}$ions is to irrigate the airways with our regular table salt at a 3-4\% hypertonic concentration. In humans, a pilot open-labelled randomised controlled trial (RCT), published in 2019, and revised for coronavirus infections in 2020, showed in adults that hypertonic saline (HS) nasal irrigation and gargling achieved a highly significant effect against common colds, caused by several viruses including coronaviruses [4-6].

Prompted by these findings, global uncertainties and the need to have safe, adjunctive practical approach like hand-washing to combat early SARS-CoV-2 infections during lockdowns [6,7], we report a successful swift response to nasal rinsing and gargling with HS in an asymptomatic SARS-CoV-2 infected physician.

\section{Case presentation}

We describe a 54-year-old asymptomatic SARS-CoV-2-positive physician, working at Bambino Gesù Children's Hospital in Rome when the Italian acute respiratory syndrome coronavirus 2 (COVID-19) pandemic began at the beginning of March 2020. A 62-year-old woman diagnosed and admitted to the Spallanzani Hospital COVID-19 Unit in Rome, Italy, for a severe COVID-19 pneumonia without needing intensive care, infected this healthy physician, moderate smoker, who had no previous history for respiratory diseases. The COVID-19 infected woman recovered in two weeks.

On March 4th 2020, the physician's nasopharyngeal swab tested by reverse transcriptase polymerase chain reaction (RT-PCR) (an assay that has high test performance because it amplifies 3-target: E, RdRP and N SARS-CoV-2 genes) [8], resulted positive (low cycle threshold

${ }^{\star}$ Correspondence to: Paola Rosati MD MSc, Clinical pathways and Epidemiology Unit, Bambino Gesù Children's Hospital, Largo S. Onofrio 4, 00165 Rome, Italy, Tel 003906 68593654; E-mail: paola.rosati@opbg.net

Key words: 2019 novel coronavirus, SARS-CoV-2, COVID-19, hypertonic saline solution, nasal rinsing and gargling, quarantine

Received: July 27, 2020; Accepted: August 08, 2020; Published: August 10, 2020 
value) for SARS-CoV-2 (Allplex ${ }^{\text {ra }}$ 2019-nCoV Assay kit - Seegene, South Korea). While in quarantine at home, the SARS-CoV-2 infected physician, according to published RCT results $[4,5]$, and information provided by the freely available link http://www.elvisstudy.com/nasalirrigation-and-gargling.html, prepared daily an HS solution by boiling water, adding table salt at the highest comfortable concentration (up to 2 teaspoonfuls in two cups of boiled water, $500 \mathrm{ml}$, about $3-4 \%$ $\mathrm{NaCl}$ ), then mixed and cooled it before irrigating his airways. $\mathrm{He}$ washed his nose with HS 6-times a day, using a disposable syringe, and gargled saline. Occasionally, he used more expensive single-use vials or spray bottles of $\mathrm{HS}$ products $(3 \% \mathrm{NaCl})$ from a pharmacy. During his quarantine at home from March, 4th 2020, the physician continued repeating daily HS nasal rinsing and gargling alone, and remained completely asymptomatic. In the supplementary file to this paper, the physician described his perceptions using HS as a burnt feeling in the mouth and nose resembling what he feels when diving into seawater when summer holidays begin. He had no other side effects. In three subsequent nasopharyngeal samples taken 3, 10, and 14 days later, the physician's SARS-CoV-2 RT-PCR test results became negative. At the end of April, after the third negative SARS-CoV-2 nasopharyngeal swab test, the physician now healthy returned to his hospital work. In May 2020, more than two months after the SARS-CoV-2 infection, his serum immunoglobulin G (IgG) for SARS-CoV-2 tested negative at Bambino Gesù Children's Hospital laboratory in Rome. The physician-patient's personal experience and perspective is available in a Supplementary file.

\section{Discussion}

No published papers have described a similar case. In these pandemic times, despite the mortality rate for the Chinese COVID-19 epidemic acknowledged worldwide $[9,10]$, several virologists, the media, Wellcome Trust and WHO websites (https://wellcome.ac.uk/ coronavirus-covid-19/open-data; https://www.who.int/emergencies/ diseases/novel-coronavirus-2019/advice-for-public/myth-busters) have overwhelmed people with information on contrasting treatment results, and retracted multinational reports, whereas clinical RCTs on costly medications and knowledge on the longevity of neutralising antibodies IgG against SARS-CoV-2 in asymptomatic individuals are still underway [11-16]. Hence, despite the limited clinical evidence available [2-5], in the current pandemic urgency state we wonder why family doctors, the media, and health authorities decline to propose washing-out SARS-CoV-2 by with HS as an adjunctive, simple, costfree airway procedure like hand-washing [6].

The available biochemical evidence [1,2] and our case report highlight how increasing $\mathrm{Cl}^{-}$ion availability by repeatedly washing the nose and throat with a cost-free HS solution at home could easily and quickly combat SARS-CoV-2 infections at an early stage, also in developing countries. In these low-income countries, people who prepare HS at home should avoid possible contamination. Health authorities must give guidance to ensure that the technique is used safely.

As Ramalingam et al. mentioned [4,5], the salt nasal irrigation and gargling technique probably also benefits SARS-CoV-2 infected individuals by reducing infectivity. Testing SARS-CoV-2 nasopharyngeal swabs, as in our case report, by RT-PCR, an assay targeting three SARS$\mathrm{CoV}-2$ antigens, has a high accuracy and test performance thus reducing the risk of false positive or false negative tests [8]. Our case report of an asymptomatic SARS-CoV-2 individual who tested negative to serum IgG against SARS-CoV-2 two months after the nasopharyngeal positive swab test tends to confirm the uncertain COVID-19 antibody longevity reported also in a recent Cochrane review [16].

In conclusion, while we await evidence from well-structured clinical trials, in the current pandemic urgency, our case report seemingly supports as a simple expedient like hand-washing inexpensive HS nasal rinsing and gargling at home to combat early SARS-CoV-2 infected individuals. A limitation is that HS irrigations remain unproven to combat multifaceted organ disorders that the COVID-19 infection might determine, including challenges in SARS-CoV-2 infection findings and critical cases [17], and the newest inflammatory multisystem syndrome temporally associated with SARS-CoV-2 in children [18]. A highly effective early technique that could be used early and cost-free during COVID-19 pandemic would nevertheless provide enormous health as well as economic benefits.

Despite the need for subjecting HS to public scrutiny in the scientific arena, health authorities could publicize HS nasal rinsing and gargling as an inexpensive, effective, procedure for early SARS-CoV-2 "wash-out" $[3,5,6]$, having as minor potential risks only a burnt feeling in the mouth and nose from an excessively salty solution [6]. Besides research on costly combined drugs for COVID-19, and the lengthy time needed before vaccines become available, well-designed RCTs could test the HS procedure in quarantined people at home, and in vulnerable populations such as the elderly and children living in developed and developing countries. Adding to the preventive lockdown policy recommendations, handwashing, masks and social distancing, this "less is more" HS practical technique might provide an easy, cost-free, adjunctive treatment option to combat early SARS-CoV-2 infection, reduce intensive care burdens, and avoid foreseeable pandemic virus outbreaks and possible second COVID-19 waves.

\section{Physician-patient's perspective}

"I was really shocked and scared when on the 4 th March I discovered that I was infected by taking care of a COVID-19 infected woman. Being asymptomatic and quarantined, I searched the Internet for evidence about possible available treatments and read the results about the Edinburgh team RCT on the hypertonic saline nasal rinsing and gargling. I immediately started the procedure, by preparing every day at home the saline solution, and occasionally using a similar product bought in the pharmacy. Hence, I diluted 2 teaspoons of table salt in $500 \mathrm{ml}$ of boiled water, irrigated my nose and gargled the solution 6 times a day with a disposable syringe before each meal, according to what I learnt from the free online Scottish research video. At every irrigation, I felt only a slight, short-lasting, bearable burning sensation in the nose and throat mucous membranes. This feeling reminded me how I feel every time I dive into seawater when summer holidays begin. I had no other side effects. To test exclusively the possible benefits of the hypertonic solution, I avoided any other treatment or medication. Hence, three days after the SARS-CoV-2 positive nasopharyngeal swab test, when I received the results of the negative second SARSCoV-2 swab, I was excited. Encouraged by the rapid result, I used the hypertonic saline procedure every day and remained asymptomatic. The confirmed negative results in two other swab tests allowed me to go back to my hospital shifts in April. In May, more than two months after the SARS-CoV-2 infection, the COVID-19 serum Ig G tested negative. In my case, I think that rapidly using hypertonic saline nasal irrigation and gargling "washed-out" the SARS-CoV-2 infection, impeded "the disease" spread, and therefore avoided the COVID-19 multi-organ inflammation, a trigger for the neutralising antibody IgG immune response and longevity. This hypothesis on why I tested negative on 
serum IgG at more than two months after the SARS-CoV-2 infection, is supported also by a recent pre-print paper from the King's College in London that showed that in SARS-CoV-2 asymptomatic individuals the IgG levels waned in a period over 40 days. During these tough pandemic times, from what I learnt and my successful experience, I wonder why everyone doesn't use this simple, practical procedure, thus defending themselves against the SARS-CoV-2 infection early at home at their own pace."

\section{Declarations}

Ethical approval and consent to participate: All information reported in this article was conducted in accordance with institutional ethical guidelines. The physician-patient gave written informed consent to participate in our research.

Competing interests: The authors report no competing interests or any conflict of interests.

Funding: The authors report no funding sources for this research and case report.

Authors' contributions: PR and UG conceived the study. CC tested the SARS-CoV-2 from nasopharyngeal swab tests and serum IgG. PR, UG wrote the manuscript and contributed equally to this article. PR, UG and CC critically reviewed the manuscript, read and approved the final version.

\section{References}

1. Wang G (2016) Chloride flux in phagocytes. Immunol Rev 273: 219-231. [Crossref]

2. Ramalingam S, Cai B, Wong J, Twomey M, Chen R, et al. (2018) Antiviral innate immune response in non-myeloid cells is augmented by chloride ions via an increase in intracellular hypochlorous acid levels. Sci Rep 8: 13630.

3. King D, Mitchell B, Williams CP, Spurling GKP (2015) Saline nasal irrigation for acute upper respiratory tract infections. Cochrane Database Syst Rev 4: CD006821. [Crossref]

4. Ramalingam S, Graham C, Dove J, Morrice L, Sheikh A (2019) A pilot, open labelled, randomised controlled trial of hypertonic saline nasal irrigation and gargling for the common cold. Sci Rep 9: 1015. [Crossref]
5. Ramalingam S, Graham C, Dove J, Morrice L, Sheikh A (2020) Hypertonic saline nasal irrigation and gargling should be considered as a treatment option for COVID-19. J Glob Health 10: 010332. [Crossref]

6. Farrell NF, Klatt-Cromwell C, Schneider JS (2020) Benefits and safety of nasal saline Irrigations in a pandemic - washing COVID-19 away. JAMA Otolaryngol Head Neck Surg.

7. Melnick ER, Ioannidis JPA (2020) Should governments continue lockdown to slow the spread of covid-19? BMJ 369: m1924.

8. Corman VM, Landt O, Kaiser M, Molenkamp R, Meijer A, et al. (2020) Detection of 2019 novel coronavirus $(2019-\mathrm{nCoV})$ by real-time RT-PCR. Euro Surveill 25: 2000045. [Crossref]

9. Feng Z, Li Q, Zhang Y, Wu Z, Dong X, et al. (2020) The epidemiological characteristics of an outbreak of 2019 novel coronavirus diseases (COVID-19) - China, 2020. The novel coronavirus pneumonia emergency response epidemiology team. China $C D C$ Weekly 8: 113-122.

10. Guzzetta G, Poletti P, Ajelli M, Trentini F, Marziano V, et al. (2020) Potential shortterm outcome of an uncontrolled COVID-19 epidemic in Lombardy, Italy, February to March 2020. Euro Surveill 25: 2000293.

11. Beigel JH, Tomashek KM, Dodd LE, Mehta AK, Zingman BS, et al. (2020) Remdesivir for the treatment of Covid-19. Preliminary report. $N$ Engl J Med.

12. Ioannidis JPA (2020) Coronavirus disease 2019: the harms of exaggerated information and non-evidence-based measures. Eur J Clin Invest 50: e13222.

13. Mehra MR, Ruschitzka F, Patel AN (2020) Retraction-Hydroxychloroquine or chloroquine with or without a macrolide for treatment of COVID-19: a multinational registry analysis [retraction of: Lancet. 2020 May]. Lancet 395: 1820.

14. Seow J, Graham C, Merrick B, Acors S, Steel KJA, et al. (2020) Longitudinal evaluation and decline of antibody responses in SARS-CoV-2 infection.

15. Zarocostas J (2020) How to fight an infodemic. Lancet 395: 676. [Crossref]

16. Deeks JJ, Dinnes J, Takwoingi Y, Davenport C, Spijker R, et al. (2020) Antibody tests for identification of current and past infection with SARS-CoV-2. Cochrane Database Syst Rev 6: CD013652. [Crossref]

17. Elkabily AG, Sebaiy MM (2020) A suggestion of using Ang-(1-7) and/or GLP-1 receptor agonists in high mortality patients with COVID-19. Trends Med 20.

18. Whittaker E, Bamford A, Kenny J, Kaforou M, Jones CE, et al. (2020) Clinical characteristics of 58 children with a pediatric inflammatory multisystem syndrome temporally associated with SARS-CoV-2. JAMA 324: 259-269.

Copyright: (C2020 Rosati P. This is an open-access article distributed under the terms of the Creative Commons Attribution License, which permits unrestricted use, distribution, and reproduction in any medium, provided the original author and source are credited. 